\title{
Non-perturbative determination of improvement coefficients using coordinate space correlators in $N_{f}=2+1$ lattice QCD
}

\author{
Piotr Korcyl* \\ Institut für Theoretische Physik, Universität Regensburg, D-93040 Regensburg, Germany \\ E-mail: piotr.korcyleur.de
}

\section{Gunnar S. Bali}

Institut für Theoretische Physik, Universität Regensburg, D-93040 Regensburg, Germany

E-mail: gunnar.baliaur.de

\begin{abstract}
We determine quark mass dependent order $a$ improvement terms of the form $b_{J} a m$ for non-singlet scalar, pseudoscalar, vector and axialvector currents, using correlators in coordinate space. We use a set of CLS ensembles comprising non-perturbatively improved Wilson Fermions and the tree-level Luescher-Weisz gauge action at $\beta=3.4,3.46,3.55$ and $\beta=3.7$, corresponding to lattice spacings $a$ ranging from $0.05 \mathrm{fm}$ to $0.09 \mathrm{fm}$. We report the values of the $b_{J}$ improvement coefficients which are proportional to non-singlet quark mass combinations and also discuss the possibility of determining the $\bar{b}_{J}$ coefficients which are proportional to the trace of the quark mass matrix.
\end{abstract}

34th annual International Symposium on Lattice Field Theory

24-30 July 2016

University of Southampton, UK

\footnotetext{
* Speaker.
} 


\section{Introduction}

Monte Carlo simulations of Quantum Chromodynamics suffer from discretizations effects. Depending on the chosen discretization of the action such effects can disappear proportionally to a power of the lattice spacing $a$. Wilson's prescription for the discrete pure gauge action gives $a^{2}$ cutoff effects, whereas the Dirac-Wilson operator introduces corrections linear in the lattice spacing $a$ due to the Wilson term. One can account for the latter following the Symanzik improvement programme. In the scaling regime the lattice action can be approximated by its continuum counterpart supplemented by corrections proportional to positive powers of the lattice spacing,

$$
S_{\mathrm{QCD}}(a(\beta))=S_{\text {continuum }}+a S_{1}+a^{2} S_{2}+\ldots
$$

The so-called $\mathscr{O}(a)$-improvement programme for Wilson fermions consists of adding to $S_{\mathrm{QCD}}$ irrelevant operators entering $S_{1}$ with numerical coefficients chosen in such a way as to remove the entire contribution of that term. Improvement of the action $S_{\mathrm{QCD}}$ requires knowledge of the $c_{\mathrm{SW}}$ coefficient and, for non-vanishing quark masses, also of $b_{g}$ and $b_{m}$. Full order $a$ improvement requires not only to improve the action but also operators. Taking the example of the non-singlet axial current, we usually define

$$
A_{\mu}^{j k, \mathrm{R}}(x)=Z_{A}\left(1+a b_{A} m_{j k}+a 3 \bar{b}_{A} \bar{m}\right)\left\{\bar{\psi}_{j}(x) \gamma_{\mu} \gamma_{5} \psi_{k}(x)+a c_{A} \partial_{\mu}^{\mathrm{sym}} P^{j k}(x)\right\}
$$

Hence, for full $\mathscr{O}(a)$-improvement of a non-singlet quark bilinear $J$, using massive Wilson fermions one needs $c_{\mathrm{SW}}, b_{g}, b_{m}, c_{J}, b_{J}, \bar{b}_{J}$. The remaining cut-off effects vanish as $a^{2}$.

In the present proceedings we summarize results obtained for the $b_{J}$ and $\tilde{b}_{J}{ }^{1}$ improvement coefficients. For more details we refer the Reader to Ref. [1]. We follow the proposal of Ref. [2] and use coordinate space correlators to construct observables sensitive to $b_{J}$ and $\tilde{b}_{J}$. In particular, we adapt the original suggestion to the case of the CLS ensembles with $N_{f}=2+1$ dynamical flavours $[3,4]$ and implement several improvements and modifications. We present results for the flavour non-singlet scalar, pseudoscalar, vector and axialvector currents. The CLS ensembles feature nonperturbatively improved Wilson fermions and the tree-level Lüscher-Weisz gauge action. $c_{\mathrm{SW}}$ was estimated in [5], whereas $c_{A}$ in [6]. We simulate at the bare inverse coupling constant values $\beta=3.4,3.46,3.55$ and 3.7, which correspond to lattice spacings $a \in[0.05,0.09] \mathrm{fm}$. The subset of CLS ensembles used in the present study is presented in table 1.

For completeness we briefly introduce some notations. We denote quark mass averages as

$$
m_{j k}=\frac{1}{2}\left(m_{j}+m_{k}\right), \quad m_{j}=\frac{1}{2 a}\left(\frac{1}{\kappa_{j}}-\frac{1}{\kappa_{\text {crit }}}\right), \quad \bar{m}=\frac{1}{3}\left(m_{s}+2 m_{\ell}\right) .
$$

with the subscripts $j, k$ corresponding to different quark content: $j, k=1,2,3=$ light, light, strange. The light quark mass $m_{\ell}$ and the average quark mass $\bar{m}$ can be used to parameterize the mass dependence of physical observables.

\footnotetext{
${ }^{1}$ Determining $\bar{b}_{J}$ requires $b_{g}$ which is currently unavailable. Instead, we determine $\tilde{b}_{J}$ which is a combination of $\bar{b}_{J}$ and $b_{g}$

$$
\tilde{b}_{J}\left(g^{2}\right)=\bar{b}_{J}\left(g^{2}\right)+\frac{b_{g}\left(g^{2}\right)}{N_{f}}\left[\frac{\partial \ln Z_{J}^{R}\left(g^{2}, a \mu\right)}{\partial g^{2}}-\frac{\gamma_{J}\left(g^{2}\right)}{4 \pi \beta\left(g^{2}\right)}\right] g^{2} .
$$

For practical purposes the knowledge of $\tilde{b}_{J}$ is sufficient. For a derivation of Eq. (1.3) and more details, see Section II of Ref. [1].
} 


\begin{tabular}{|c||c|c|c|c|c|}
\hline$\beta$ & name & $\kappa_{l}$ & $\kappa_{s}$ & \# conf. & step \\
\hline 3.4 & H101 & 0.136759 & 0.136759 & 100 & 40 \\
3.4 & H102 & 0.136865 & 0.136549339 & 100 & 40 \\
3.4 & H105 & 0.136970 & 0.13634079 & 103 & 20 \\
3.4 & H106 & 0.137016 & 0.136148704 & 57 & 20 \\
3.4 & H107 & 0.136946 & 0.136203165 & 49 & 20 \\
3.4 & C101 & 0.137030 & 0.136222041 & 59 & 40 \\
3.4 & C102 & 0.137051 & 0.136129063 & 48 & 40 \\
3.4 & rqcd17 & 0.1368650 & 0.1368650 & 150 & 40 \\
3.4 & rqcd19 & 0.13660 & 0.13660 & 50 & 40 \\
\hline 3.46 & S400 & 0.136984 & 0.136702387 & 83 & 40 \\
\hline 3.55 & N203 & 0.137080 & 0.136840284 & 74 & 40 \\
\hline 3.7 & J303 & 0.137123 & 0.1367546608 & 38 & 40 \\
\hline
\end{tabular}

Table 1: List of CLS ensembles used in the study. For details see Ref. [3, 4]. The number of independent measurements for each ensemble is given in column 5. "step" denotes the spacing in MDU between consecutive measurements. Such spacing guarantees that autocorrelations are negligible.

Our primary observable are the connected Euclidean current-current correlation functions. We denote their continuum, renormalized in the scheme $R$ at a scale $\mu$, (where, for example, $R=\overline{\mathrm{MS}}$ ), versions as

$$
G_{J^{(j k)}}^{R}\left(x, m_{\ell}, m_{s} ; \mu\right)=\left\langle\Omega\left|T J^{(j k)}(x) \bar{J}^{(j k)}(0)\right| \Omega\right\rangle^{R}
$$

\section{Method}

We start with the following two observations valid for correlation functions at distances $x^{2} \ll$ $1 / \Lambda_{\mathrm{QCD}}^{2}$ when $m^{2}<\Lambda_{\mathrm{QCD}}^{2}[2,1]$.

1. The continuum correlation function differs from that of the massless case by mass dependent terms

$$
G_{J^{(j k)}}^{R}\left(x, m_{\ell}, m_{s} ; \mu\right)=G_{J^{(j k)}}^{R}(x, 0,0 ; \mu) \times\left[1+\mathscr{O}\left(m^{2} x^{2}, m^{2}\langle F F\rangle x^{6}, m\langle\bar{\psi} \psi\rangle x^{4}, m\langle\bar{\psi} \sigma F \psi\rangle x^{6}\right)\right],
$$

2. The continuum flavour non-singlet Green function $G^{R}$ above can be related to the corresponding Green function $G$ obtained in the lattice scheme at a lattice spacing $a=a\left(g^{2}\right)$ as follows:

$$
G_{J^{(j k)}}^{R}\left(x, m_{\ell}, m_{s} ; \mu\right)=\left(Z_{J}^{R}\right)^{2}\left(g^{2}, a \mu\right) \times\left(1+2 b_{J} a m_{j k}+6 \tilde{b}_{J} a \bar{m}\right) G_{J^{(j k), I}}\left(n, a m_{j k}, a \bar{m} ; g^{2}\right),
$$

Note that we replaced $Z^{R}\left(\tilde{g}^{2}\right)$ by $Z^{R}\left(g^{2}\right)$ and absorbed the difference, replacing $\bar{b}_{J}$ by $\tilde{b}_{J}$, see Eq. (1.3). Hence, by constructing a ratio of two correlation functions with identical Dirac structure we can eliminate the massless correlation function on the right-hand side of Eq. (2.1) and the mass independent renormalization constant in Eq. (2.2). We arrive at the following expression 


$$
\frac{G_{J^{(j k)}}\left(n, a m_{j k}^{(\rho)}, a \bar{m}^{(\rho)} ; g^{2}\right)}{G_{J^{(r s)}}\left(n, a m_{r s}^{(\sigma)}, a \bar{m}^{(\sigma)} ; g^{2}\right)}=1+2 b_{J} a\left(m_{r s}^{(\sigma)}-m_{j k}^{(\rho)}\right)+6 \tilde{b}_{J} a\left(\bar{m}^{(\sigma)}-\bar{m}^{(\rho)}\right)+\mathscr{O}\left(a^{2}, x^{2}\right),
$$

where the $\rho$ and $\sigma$ indices distinguish different points in the $\kappa_{\ell}-\kappa_{s}$ parameter plane. We now define two observables which are directly proportional to the improvement coefficients we are after. In the first case we choose one ensemble away from the symmetric line, i.e. $m_{\ell} \neq m_{s}$, and measure two correlation functions: one with two light quarks and the second with a light and a strange quark. Their ratio is approximated by

$$
R_{J}\left(x, m_{12}^{(\rho)}, m_{13}^{(\rho)}\right) \equiv \frac{G_{J^{(12)}}\left(n, a m_{12}^{(\rho)}, a \bar{m}^{(\rho)} ; g^{2}\right)}{G_{J^{(13)}}\left(n, a m_{13}^{(\rho)}, a \bar{m}^{(\rho)} ; g^{2}\right)}=1+2 b_{J} a\left(m_{13}^{(\rho)}-m_{12}^{(\rho)}\right)=1+2 b_{J} a \delta m
$$

Note that $\kappa_{\text {crit }}$ as well as $\tilde{b}_{J}$ cancel. For the second observable we choose two ensembles on the symmetric line and we define $\widetilde{R}_{J}\left(x, \delta \bar{m}=\bar{m}^{(\sigma)}-\bar{m}^{(\rho)}\right)$

$$
\widetilde{R}_{J}\left(x, \bar{m}^{(\sigma)}, \bar{m}^{(\rho)}\right) \equiv \frac{G_{J^{(12)}}\left(n, a \bar{m}^{(\rho)}, a \bar{m}^{(\rho)} ; g^{2}\right)}{G_{J^{(12)}}\left(n, a \bar{m}^{(\sigma)}, a \bar{m}^{(\sigma)} ; g^{2}\right)}=1+\left(2 b_{J}+6 \tilde{b}_{J}\right) a\left(\bar{m}^{(\sigma)}-\bar{m}^{(\rho)}\right)=\widetilde{R}_{J}(x, \delta \bar{m}),
$$

which gives us sensitivity to $\tilde{b}_{J}$ once $b_{J}$ is known. Again, no knowledge of $\kappa_{\text {crit }}$ is needed. Figure 1 shows $R_{J}(x, \delta m)$ and $\widetilde{R}_{J}(x, \delta \bar{m})$ as functions of $\delta m$ and $\delta \bar{m}$ for a given vector $n$. A linear behaviour of the data confirms the validity of expressions Eq. (2.4) and Eq. (2.5).
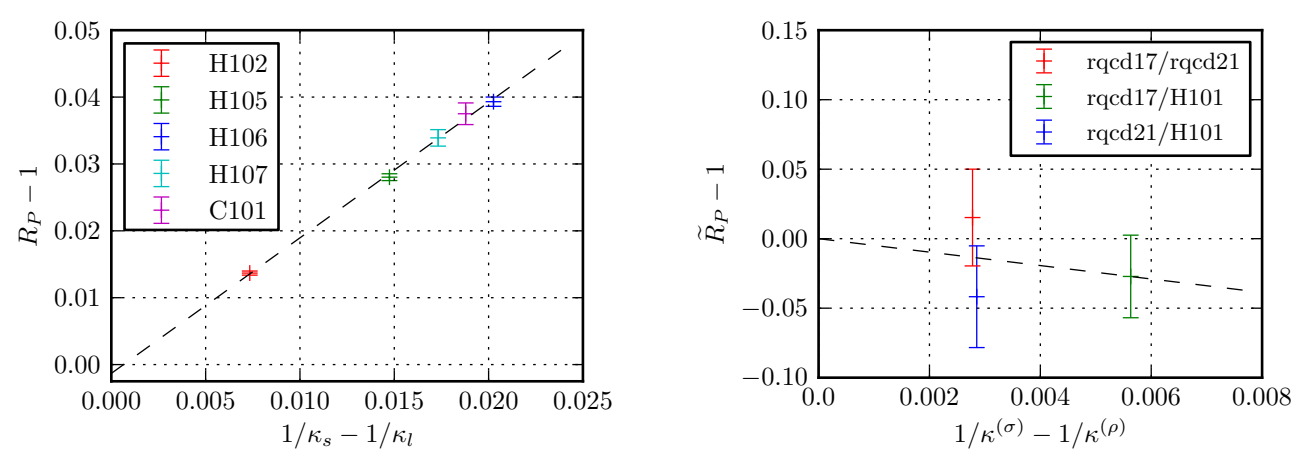

Figure 1: Example of data in the pseudoscalar channel for a given vector $n$. The quantity plotted is $R_{P}-1$ for $n=(0,1,1,1)$ on the left and $\widetilde{R}_{P}-1$ for $n=(0,1,2,2)$ on the right against quark mass difference. A linear dependence is expected, with a slope corresponding to the improvement coefficient $b_{P}$ and $\bar{b}_{P}$.

\section{Short and medium distance corrections}

The observables $R_{J}(x, \delta m)$ and $\widetilde{R}_{J}(x, \delta \bar{m})$ are affected by undesired cut-off effects which are mostly visible at short distances $(<0.2 \mathrm{fm})$ as well as mass dependent non-perturbative corrections relevant at medium distances $(\approx 0.3-0.4 \mathrm{fm})$. We compensate for both of them at tree-level 
perturbation theory and propose improved observables $B_{J}(x, \delta m)$ and $\widetilde{B}_{J}(x, \delta \bar{m})$ with these effects subtracted.

Figure 2 shows the observable $R_{J}(x, \delta m)$ computed in tree-level lattice perturbation theory. The continuum tree-level prediction is $b_{J}^{\text {tree }}=1$, hence all deviations from that value on figure 2 are due to non-leading lattice artifacts. Using these data we can construct a set of distances $x$ for which cut-off effects are minimal. Subsequently, we only consider vectors for which the tree-level cut-off effects are smaller than $15 \%$. The improved observables $B_{J}$ and $\widetilde{B}_{J}$ have these corrections subtracted.
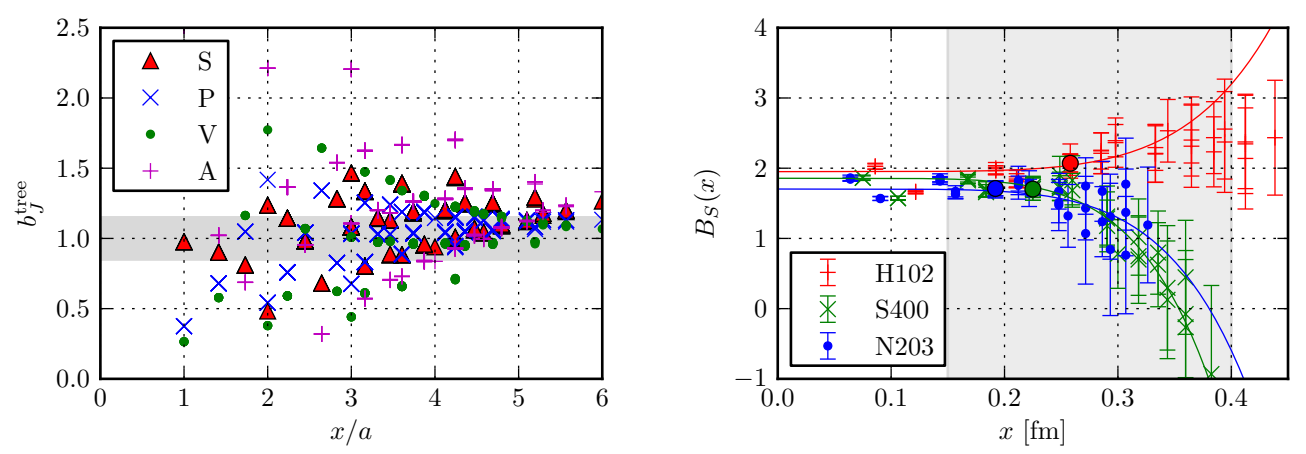

Figure 2: Left panel: Observables $R_{J}$ estimated in tree-level lattice perturbation theory plotted against the expected continuum prediction $b_{J}^{\text {tree }}=1$. In the analysis we use only vectors for which the tree-level cutoff artifacts are smaller than $15 \%$, i.e. which lie within the shaded band. Right panel: Data for $b_{S}$ for all investigated vectors $n$ for three ensembles: H102, S400 and N203. The shaded area corresponds to the interval of $x$ used in the fit which estimates the size of $x^{6}$ corrections.

At medium distances we can predict the behaviour of our data as a function of $x$ using the Operator Product Expansion. For the ratio of correlation functions one gets

$$
\frac{G_{J^{(12)}}(x)}{G_{J^{(34)}}(x)}=1+\left(A_{12}^{J}-A_{34}^{J}\right) x^{2}+\left[\left(A_{34}^{J}\right)^{2}-A_{12}^{J} A_{34}^{J}+B_{12}^{J}-B_{34}^{J}\right] x^{4}+\cdots,
$$

with the mass dependent coefficients

$$
A_{j k}^{J}=-\frac{1}{4}\left(m_{j}^{2}+m_{k}^{2}+\frac{m_{j} m_{k}}{s_{J}}\right), \quad B_{j k}^{J}=\frac{\pi^{2}}{32 N}\langle F F\rangle+\frac{m_{j}^{2} m_{k}^{2}}{16}+\frac{\pi^{2}}{8 N} \frac{2+s_{J}}{s_{J}}\left(m_{j}+m_{k}\right)\langle\bar{\psi} \psi\rangle .
$$

where $s_{J}$ are constants and depend on the Dirac structure, $s_{S}=1=-s_{P}$ and $s_{A}=\frac{1}{2}=-s_{V}$. Knowing the explicit form of these corrections and using the Gell-Mann-Oakes-Renner relation $\left(m_{j}+m_{k}\right)\langle\bar{\psi} \psi\rangle=-F_{0}^{2} M_{j k}^{2}$, where $M_{j k}$ denotes the mass of a pseudoscalar meson composed of (anti)quarks of masses $m_{j}$ and $m_{k}$ and the pion decay constant in the $N_{f}=3$ chiral limit reads $F_{0}=86.5(1.2) \mathrm{MeV}[7,8]$, we correct our observables $R_{J}$ and $\widetilde{R}_{J}$ by subtracting the leading continuum corrections. 
Hence, we arrive at the improved observable

$$
\begin{aligned}
B_{J}(x, \delta m) & \equiv\left[R_{J}(x, \delta m)-R_{J}^{\mathrm{tree}}(x, \delta m)+\frac{\pi^{2}}{8 N} \frac{2+s_{J}}{s_{J}}\left(M_{\pi}^{2}-M_{K}^{2}\right) F_{0}^{2} x^{4}\right] \times\left(\frac{1}{\kappa_{s}}-\frac{1}{\kappa_{\ell}}\right)^{-1} \\
& =b_{J}+\mathscr{O}\left(x^{6}\right)+\mathscr{O}\left(g^{2} a^{2}\right)+\cdots,
\end{aligned}
$$

and a similar expression for $\widetilde{B}_{J}(x, \delta \bar{m})$.

Figure 2 shows the numerical data for the improved observable in the scalar channel $B_{S}$. We see that the data are rather flat up until $\approx 0.3 \mathrm{fm}$. The remaining curvature is due to higher order corrections. We take them into account by attributing a systematic uncertainty to our results which we define as the size of the contribution proportional to $x^{6}$. We estimate the latter by fitting to a function containing a term $x^{6}$ within an interval of $0.15-0.4 \mathrm{fm}$.

Results in the scalar channel from all investigated ensembles are summarized in figure 3 . We note that within one value of the coupling constant the results are compatible within their errors and that the value of the $b_{S}$ improvement coefficient systematically decreases with a decreasing coupling constant, i.e. increasing $\beta$.
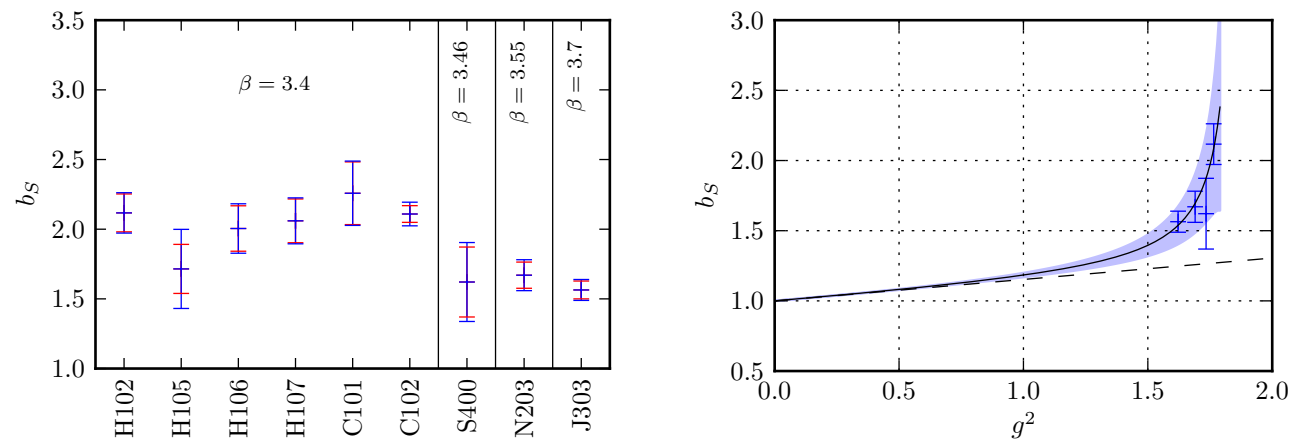

Figure 3: Left panel: Results for $b_{S}$ for all ensembles. Blue error bars correspond to the total uncertainty with the combined systematic uncertainties of a $20 \%$ uncertainty of the Wilson coefficient of the non-perturbative $x^{4}$ correction and an estimate of the size of order $x^{6}$ corrections. Red error bars correspond to the statistical uncertainty only. Final results are computed using the vector $n=(0,1,2,2)$ and all its symmetric equivalents. Right panel: Rational parametrization of $b_{S}$ as a function of the coupling constant $g^{2}$. The dashed line is the one-loop perturbative expectation.

\section{Rational parameterizations}

We present our final results for $b_{J}$ in the form of rational parameterizations. We choose four ensembles: H102, S400, N203 and J303 at four values of the coupling constant and perform a fit with the following ansatz $b_{J}\left(g^{2}\right)=1+b_{J}^{\text {one-loop }} g^{2}\left(1+\gamma_{J} g^{2}\right)\left(1+\delta_{J} g^{2}\right)^{-1}$, with $\delta_{V}=0$. The final expressions read

$$
\begin{gathered}
b_{S}\left(g^{2}\right)=1+0.11444(1) C_{F} g^{2}\left(1-0.439(50) g^{2}\right)\left(1-0.535(14) g^{2}\right)^{-1}, \\
b_{P}\left(g^{2}\right)=1+0.0890(1) C_{F} g^{2}\left(1-0.354(54) g^{2}\right)\left(1-0.540(11) g^{2}\right)^{-1}, \\
b_{V}\left(g^{2}\right)=1+0.0886(1) C_{F} g^{2}\left(1+0.596(111) g^{2}\right)
\end{gathered}
$$




$$
b_{A}\left(g^{2}\right)=1+0.0881(1) C_{F} g^{2}\left(1-0.523(33) g^{2}\right)\left(1-0.554(10) g^{2}\right)^{-1},
$$

and the fit for the case of the scalar channel is shown in figure 3. The one-loop values of the improvement coefficients for the discussed lattice action can be obtained from the results of Ref.[9].

\section{5. $\tilde{b}_{J}$ improvement coefficients}

At $\beta=3.4$ several ensembles from the symmetric line are available. We used them to test our method to extract the $\tilde{b}_{J}$ improvement coefficients. Since the ratio is composed of correlation functions measured on two different ensembles the statistical noise does not cancel and the statistics used so far yield very imprecise results:

$$
\tilde{b}_{S}=2.0(1.3)(0.3), \tilde{b}_{P}=-3.4(1.3)(0.6), \tilde{b}_{V}=-0.1(0.4)(0.1), \tilde{b}_{A}=1.4(0.4)(0.9),
$$

the first uncertainty being systematic and the second statistical. Several ways are being tested in order to improve the precision, including stochastic multiple point sources similar to the ones used in Ref. [10] or the Truncated Solver Method of Ref. [11].

\section{Conclusions}

We implemented and tested a coordinate space method to determine improvement coefficients accompanying quark mass dependent terms. The method is general and allows for the determination of improvement coefficients for all quark bilinears. With a negligible numerical effort we can achieve a $5 \%-10 \%$ precision on $b_{J}$. Improvement coefficients proportional to the trace of the mass matrix are accessible, but need a better statistical precision. These promising results encourage further steps such as the determination of the improvement coefficients $c_{J}$ or improvement coefficients for singlet fermion bilinear operators, which are of relevance in studies of the structure of nucleons, or of more complicated currents with derivatives.

\section{References}

[1] P. Korcyl, G. Bali, (2016), arXiv:1607.07090 [hep-lat].

[2] G. Martinelli et al., Phys. Lett. B 411, 141 (1997).

[3] M. Bruno et al. (CLS), J. High Energy Phys. 02, 043 (2015), arXiv:1411.3982 [hep-lat].

[4] G. Bali, E. E. Scholz, J. Simeth, W. Söldner, (2016), arXiv:1606.09039 [hep-lat].

[5] J. Bulava, S. Schaefer, Nucl. Phys. B 874, 188 (2013), arXiv:1304.7093 [hep-lat].

[6] J. Bulava, M. Della Morte, J. Heitger, C. Wittemeier (ALPHA Collaboration), Nucl. Phys. B 896, 555 (2015), arXiv:1502.04999 [hep-lat].

[7] Sinya Aoki et al. (FLAG), (2016), arXiv:1607.00299 [hep-lat].

[8] Keith A. Olive et al. (Particle Data Group), Chin. Phys. C 38, 090001 (2014).

[9] Y. Taniguchi, A. Ukawa, Phys. Rev. D 58, 114503 (1998), arXiv:hep-lat/9806015 [hep-lat].

[10] P. Korcyl, C. Lehner, T. Ishikawa, PoS LATTICE2015 (2016) 254, arXiv:1512.00069 [hep-lat].

[11] G. Bali, S. Collins, A. Schaefer, Comput.Phys.Commun.181:1570-1583 (2010), arXiv:0910.3970 [hep-lat] 